\title{
S. Landowne
}

Physics Division, Argonne National Laboratory

Argonne, IL 60439-4843 USA

CONF-8806167--4

DE89 003638

\section{INTRODUCTION}

The study of sub-barrier reactions has developed in recent years to include a broz 1 range of interconnected phenomena. The initial discuveries of enhanced sub-barrier fusion cross sections and the attempts to understand them in terms of couplings to other reaction channels have stimulated investigations into all aspects of heavy-ion collisions at low bombarding energies. As a result, new effects have been observed and new ideas are being put forward. The present talk gives an overview of some current themes in this field.

Perhaps the most encompassing development is that one can no longer think of the low energy fusion reaction as an isolated phenomenon, since the fusion rates depend crucially on the presence of other reaction channels. Thus, one wants to know what are the properties of these channels and then how to use this knowledge to explain the fusion rates quantitatively. Generally speaking, quite a number of sub-barrier fusion reactions have been measured [1] but the other reactions which occur have been isolated in a relatively few cases. It is also generally true that theoretical sub-barrier fusion calculations have been successful for a limited range of fairly light mass systems. Thus, the field continues to be a challenging area of research.

Going beyond the problem of understanding the fusion mechanism, there have been interesting new developments in all of the reaction classes that have been studied at subbarrier energies, namely, elastic scattering, inelastic excitation, transfer reactions and deep inelastic collisions. A brief discussion of each of these subjects and how they relate to fusion will be given below. In addition, the important subject of compound nuclear spin distributions produced in fusion reactions will be noted.

\section{POLARIZATION POTENTIAL FOR ELASTIC SCATTERING}

The observation of a marked energy dependence in low-energy heavy-ion elastic scattering has been an important developmen.. It has been found that the real part of the nuclear optical potential increases in strength and almost doubles as the bombarding energy is lowered toward the barrier, while the corresponding imaginary potential decreases abruptly [2]. The correlation between the energy dependence of the real "polarization" potential and the absorption can be understood in general terms by a dispersion relation [3]. A recent case of $\mathrm{Si}+\mathrm{Ni}$ is shown in Figure 1.

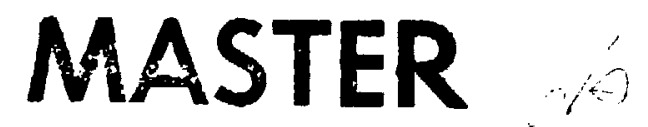

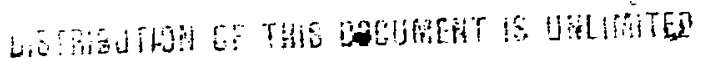




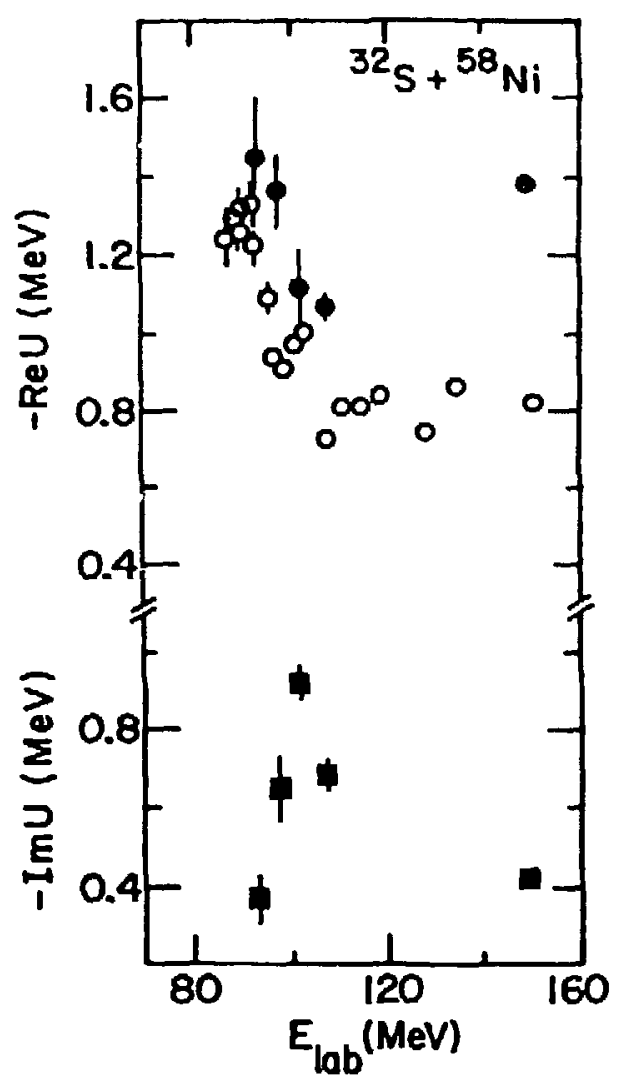

Figure 1. Effective potential strengths extracted from elastic scattering data (full points) and from fusion measurements (open points). Ref. [4]

The polarization potential is caused by the couplings of the elastic channel to other reaction channels. An expression for the effective potential can be found by treating the couplings in second-order time-dependent perturbation theory [5]. The result is

$$
\Delta U_{00}(r)=-i \sum_{\gamma} \frac{\left|V_{\gamma 0}(r)\right|^{2}}{\hbar T} \sqrt{2 / \pi} \int_{-\infty}^{\infty} d t \exp \left(-\frac{t^{2}}{\tau^{2}}-i \gamma^{t}\right) \int_{-\infty}^{d} d t^{\prime} \exp \left(-\frac{t^{\prime 2}}{\tau^{2}}+i \omega_{\gamma^{\prime}}\right)
$$

This expresses the effective potential in terms of the coupling interactions $V_{\gamma_{0}}$, the channel excitation energies $h w_{\gamma}$ and the collision time $\tau$. The real part of $\Delta U_{00}$ is the polarization potential and the imaginary part is the absorption.

There are two limiting cases for $\Delta U_{00}$. When the excitation frequencies are small compared to the inverse of the collision time one has

$$
\Delta U_{00}(r)+-i \sum_{\gamma} \frac{\left|v_{\gamma 0}(r)\right|^{2}}{\hbar / \tau} \sqrt{\pi / 2}, \quad w_{\gamma} \ll 1 / \tau
$$

which is purely absorptive. The other extreme is for high frequencies or long collision times 


$$
\Delta U_{00}(r) \cdots-\sum_{\gamma} \frac{\left|V_{\gamma 0}(r)\right|^{2}}{\hbar w_{\gamma}}, \quad w_{\gamma} \gg 1 / \tau
$$

in which case, $\Delta U_{00}$ is purely real.

\section{EFFECTIVE COUPLING FOR INELASTIC EXCITATION}

The expression for the effective potential can be generalized to give an effective coupling interaction for transitions out of the elastic channel [6],

$$
\Delta U_{\beta 0}=-i \sum_{\gamma} \frac{v_{\beta \gamma}(r) v_{\gamma 0}(r)}{\hbar \tau} \sqrt{2 / \pi} \int_{-\infty}^{\infty} d t \exp \left(-\frac{t^{2}}{\tau^{2}}+i w_{\beta \gamma} t\right) \int_{-\infty}^{t} d t^{\prime} \exp \left(-\frac{t^{\prime 2}}{\tau^{2}}+i w_{\gamma 0^{\prime}} t^{\prime}\right)
$$

Thus one expects to find similar energy dependent effects in transitions to excited states. Indeed, low energy inelastic scattering offers an important way to study such phenomena because it is sensitive to Coulomb-nuclear interferences [7].

An analysis of low-energy $\mathrm{O}+\mathrm{Pb}$ inelastic scattering is shown in Figure 2. The different curves show how the interaction must be increased to fit the data at the low energies. Additional data for this case has recently been analyzed and has confirmed that there is a large energy dependent effect similar to what has been observed for the elastic scattering [8].

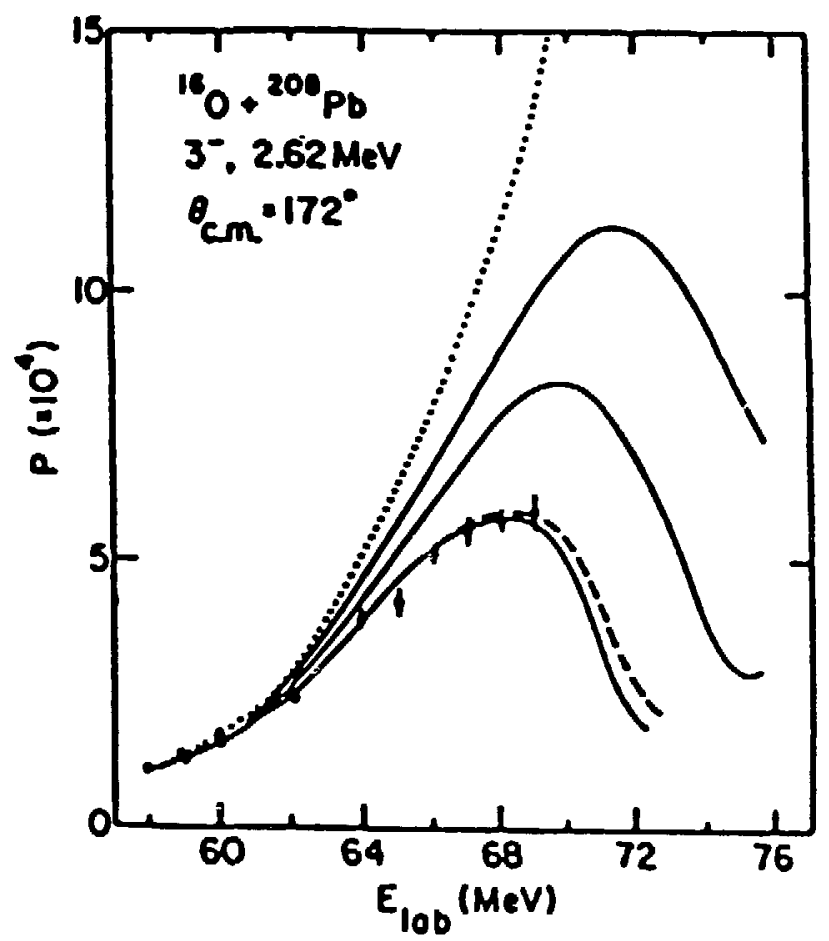

Figure 2. Low energy inelastic excitation probability [7]. 


\section{CONNECTION TO SUB-BARRIER FUSIOF}

Inasmuch as the couplings to other channels cause the energy dependence in the elastic optical potential and in the inelastic effective interaction, these effects are correlated with the enhancements in sub-barrier fusion reactions. Recently there has been an attempt to make a direct connection between these phenomena. Namely, it has been suggested that one can take the energy-dependent real potential which results from optical model analyses of elastic scattering data and use it in one-dimensional barrier penetration calculation to obtain the sub-barrier fusion [9]. Some apparent support for this idea is indicated by the analysis shown in Figure 1 . Here energy dependent potentials used to fit sub-barrier fusion cross sections are compared to the ones used to fit elastic scattering dats. There is, however, a counter-example which will be presented below.

Independently of examples for or against describing fusion in terms of an energydependent barrier, it should be noted that the fusion process involves many channels of the interacting nuclei at short distances, while an optical model analysis of elastic scattering only determines the wave function in one channel at large distances. Thus, one should not expect to find a strong correlation at the phenomenological level.

Theoretically, the only limit in which the full solution of the many-channel scattering problem can be reduced to an effective one-channel calculation is the adiabatic one where the excitation frequencies of the various channels are all large compared to the collision frequency. As indicated above by Eq. (3), the real potential in the elastic channel is renormalized by the couplings and no flux goes into the excited states. However, in this limit the polarization potential is independent of the bombarding energy (collision time). Thus, an energy dependent real potential in the elastic channel and the appearance of an absorptive potential are signals that the full problern cannot be mapped into an effective one-channel calculation.

It is also important to consider the sudden limit where the excitation frequencies are small compared to the collision frequency. In this case the coupled-channels barrier penetration problem reduces to taking averages over a distribution of one-dimensional barriers [10]. A familiar and realistic example is the case involving a deformed nucleus where one averages over the intrinsic orientations [11]. This averaging c.ver a distribution of barrier penetration factors cannot be reconciled with penetrating a single, energy-dependent barrier.

\section{SPIN-DISTRIBUTIONS}

The counter-example of the energy-dependent barrier idea mentioned above is provided by the fusion and spin-distribution measurements reported recently in Ref. [12]. These data and their analyses are reproduced in Figure $\mathbf{3}$. They also present a particular challenge since they are not well reproduced by the types of coupled-channels calculations which have been done so far. Of course, the fusion cross sections can be fit 
by using an energy-dependent potential. However, as the open circles show, the corresponding average spins do not describe the measurements.

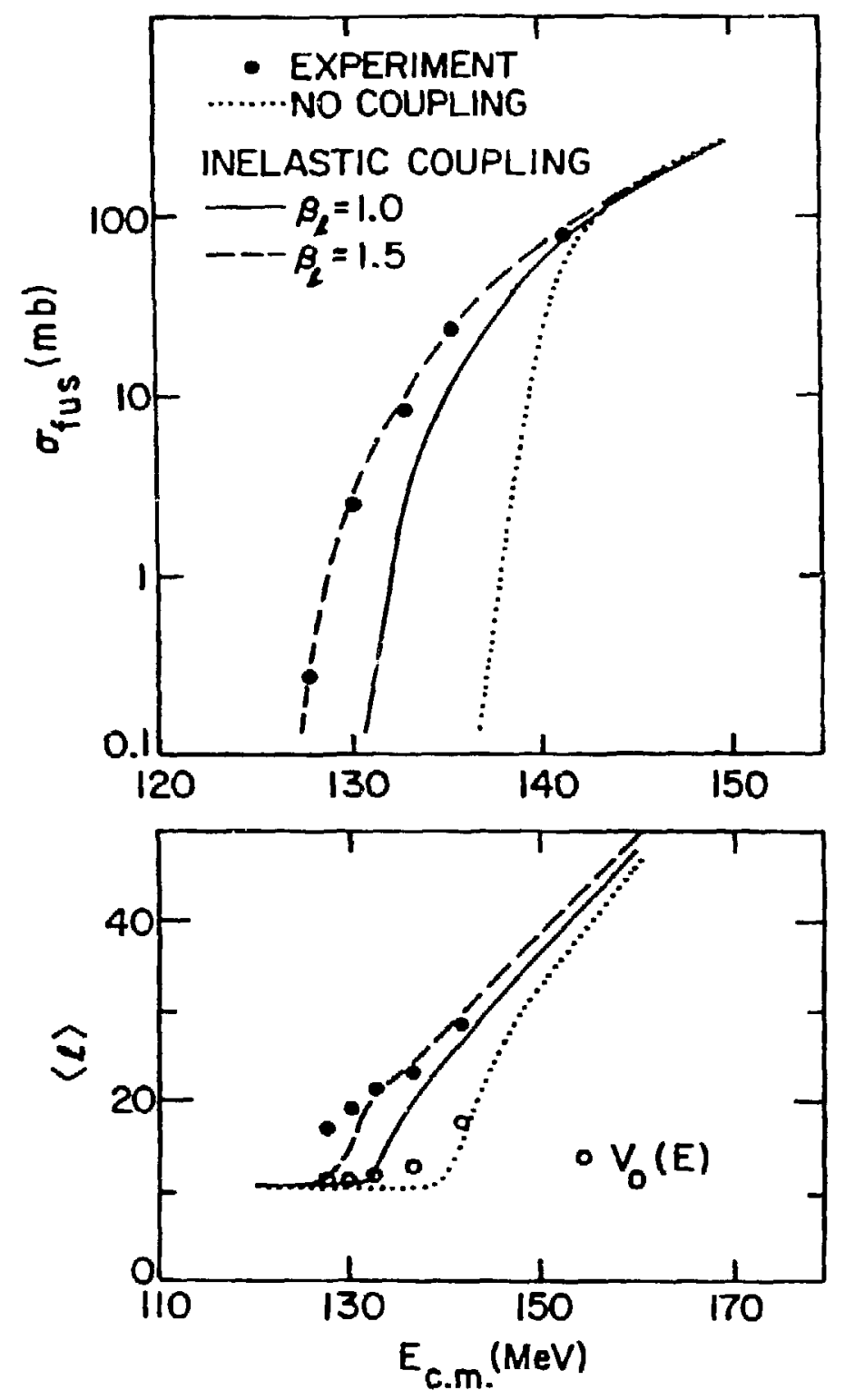

Figure 3. Fusion cross sections and average compound spins for ${ }^{64} \mathrm{Ni}+{ }^{100} \mathrm{Mo}$. Ref. [12].

The dashed curves in Figure 3 were obtained with a coupled-channels calculation using abnormally large coupling interaction strengths. The resulting spin distributions describe the data rather - $\cdot$ ell, except at the lowest two energy points. This discrepancy is significant since one expects that the energy dependence of the cross section determines the spin-distribution.

In particular, the mean-square-value of the compound nucleus epin-distribution at the energy $E$ should be related to the fusion cross section $\sigma_{f}(E)$ by [13]

$$
\left\langle\ell^{2}\right\rangle_{B}=\frac{2 \mu r_{b}^{2}}{\hbar^{2}} \frac{1}{E \sigma_{f}\left(B^{(B)}\right.} \int_{-\infty}^{B} d B^{\prime} B^{\prime} \sigma_{f}\left(B^{\prime}\right) \text {. }
$$


This relation is based on the following assumptions: (i) the fusion cross section corresponds to the flux transmitted by a distribution of barriers which are independent of $E_{\text {; }}$ (ii) the role of the partial wave angular momentum $\ell$ is essentially to increase the barrier heights by an amount $\hbar^{2} \ell^{2} / 2 \mu \mathrm{r}_{\mathrm{b}}^{2}$, where the moment of inertia at the barrier, $\mu \mathrm{r}_{\mathrm{b}}^{2}$, is assumed to be independent of $\ell$ and $E$; (iii) usually $\mu$ and $r_{b}$ are taken to be the asymptotic reduced mass and unperturbed barrier radius. These assumptions are incorporated in the calculations shown by the curves in Figure 3.

The disagreement with the average spin at the lowest energies does not necessarily mean that these assumptions are breaking down. An aiternative conclusion would be that the fusion cross section at lower energies are actually larger than the continuation of the model fit shown by the dashed curve. That is to say, the lower energy contributions to the integral in Eq. (5) have not been measured.

It should also be emphasized that the present undersianding of the compound nuclear spin distributions is not clear. The case of ${ }^{16} \mathrm{O}+{ }^{232} \mathrm{Th}$ fusion reported in Ref. [14] is an important example. An analysis of this data is shown in Figure 4.
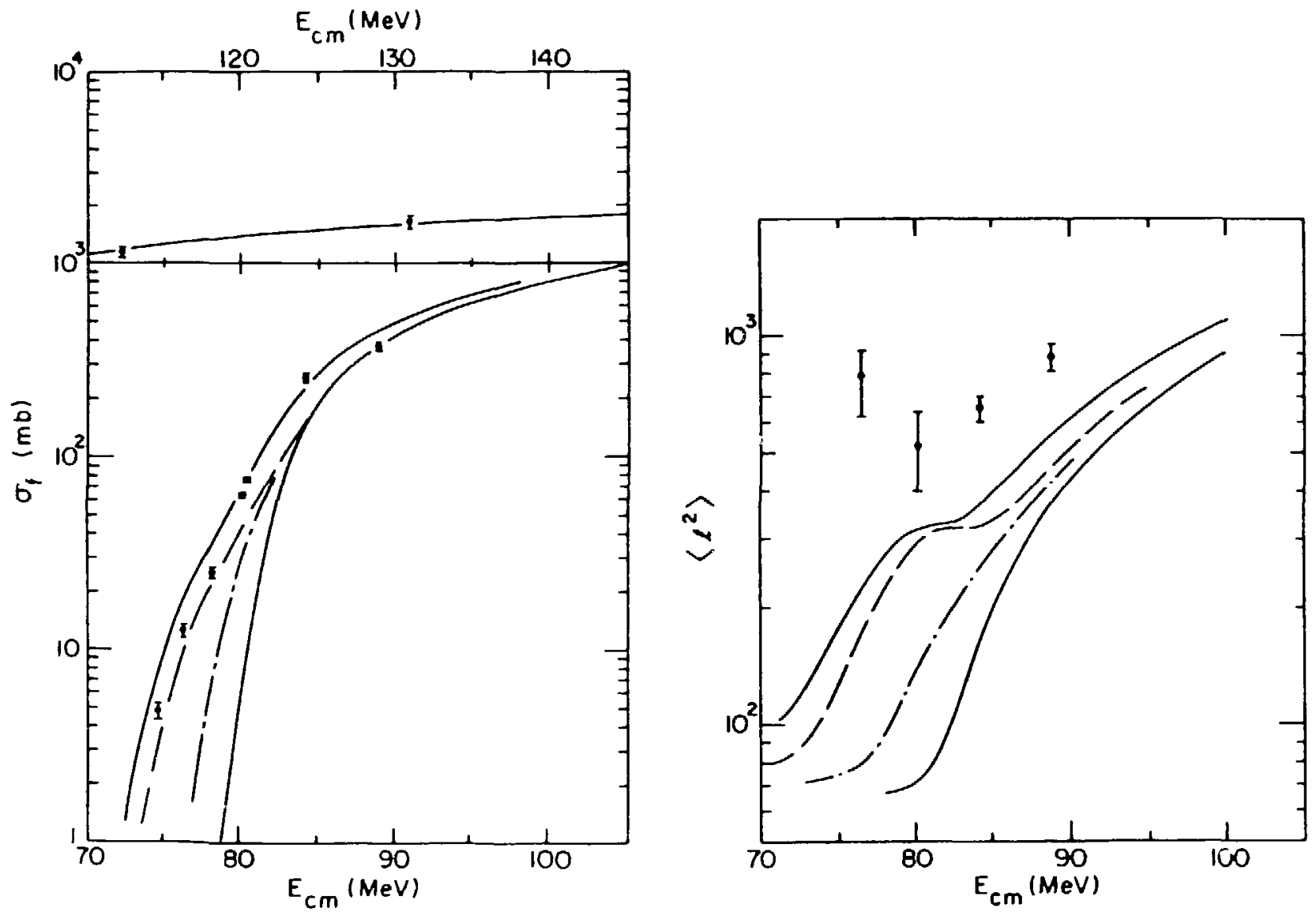

Figure 4. Fusion cross sections and average compound nuclear spins for ${ }^{16} \mathrm{O}+{ }^{232} \mathrm{Th}$.
Ref. [15] 
The calculations allow for the static quadrupole and hexadecpole moments of ${ }^{232} \mathrm{Th}$ as well as an octupole vibration [15]. They are able to account for the total fusion cross sections sather well, but the corresponding average spin values are not reproduced. It is interesting to note that the disagreement is pronounced even for the highest energy data point. This corresponds to a large total fusion cross section that is not very sensitive to the coupling effects.

\section{FUSION AND ROTATIONAL/VIBRATIONAL EXCITATION}

The previous case of ${ }^{16} \mathrm{O}+{ }^{232} \mathrm{Th}$ shown in Figure 4 is a good example of how the fusion cross section is influenced by rotational excitation. Because of the low excitation energies associated with the rotation, the coupled-channels calculation can be well approximated by averaging over the cross sections calculated for fixed orientations of the deformed surface [15]. This procedure accounts for all possible rotational states.

In other cases, like $\mathrm{Ni}+\mathrm{Ni}$, the vibrational model has been used to describe the couplings to the lowest states of a given angular momentum and parity. Here the potential radius is allowed to vibrate and a first-coder expansion is made to obtain the transition form factors. Usually the expansion is not carried out further, with the result that only independent one-phonon states of the projectile and target are allowed to couple with the ground-state entrance channel.

A recent calcralation of the $\mathrm{Ni}+\mathrm{Ni}$ case has studied the effect of taking the vibrational model to the next higher order [16]. Thus, in the expansion of the nuclear potential there appear quadratic terms in the surface amplitudes in addition to the usual linear ones. Their effect on the sub-barrier fusion was found to be substantial, as can be seen in Figure 5.

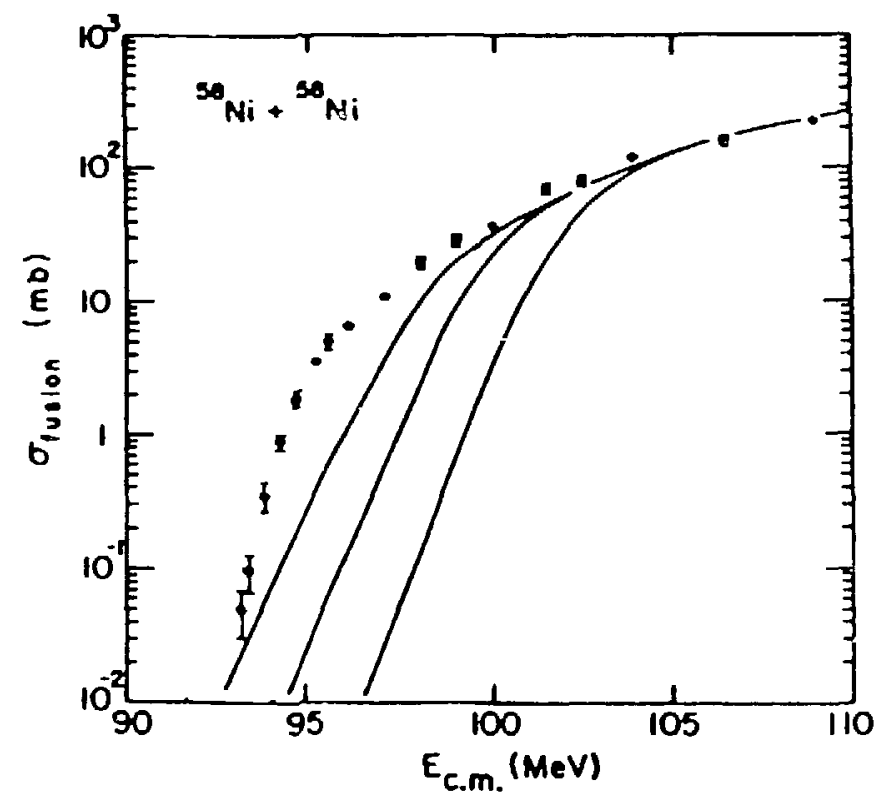

Figure 5. Fusion calculations for ${ }^{58}{ }^{-1 i}{ }^{6}+{ }^{68} \mathrm{Ni}$ allowing for first- and second-order vibrational couplings [16]. 
These second-order calculations bring in the mutual excitation process where the projectile and target are simultaneously excited. This is found to account for about half of the difference with respect to the first-order calculation shown in Figure 5. Most of the remainder comes from the fact that the second-order terms have diagonal matrix elements whici lower the potential barriers in the excited states. The ability to directly populate two-phonon states in the projectile or target does not play a strong role in these calculations. The relatively high energy of the phonons in this case is the reason why, and it also causes the third-order effects to be relatively small [16].

\section{FUSION AND TRANSFER REACTIONS}

Transfer reaction channels are not included in the calculations shown in Figure 5. It is generally believed that they should also play a role, particularly in explaining the differences between similar systems, such as ${ }^{58} \mathrm{Ni}+{ }^{64} \mathrm{Ni}$ as compared to ${ }^{58} \mathrm{Ni}+{ }^{58} \mathrm{Ni}$. Measurements for ${ }^{58} \mathrm{Ni}+{ }^{64} \mathrm{Ni}$ transfer cross sections have been carried out [17]. It is a challenge for the theory to incorporate these channels consistently with the higher-order vibrations.

A recent study has been made in this direction [18]. It incorporates an idea, based on a macroscopic point of view, that the transfer coupling interaction should depend on the distance between the nuclear surfaces which are allowed to vibrate in the same way as for the nuclear potential. Transfer form factors were parameterized in this way and were adjusted to simultaneously fit the quasi-elastic and one-particle transfer data for ${ }^{58} \mathrm{Ni}+{ }^{64} \mathrm{Ni}$, as shown in Figure 6.

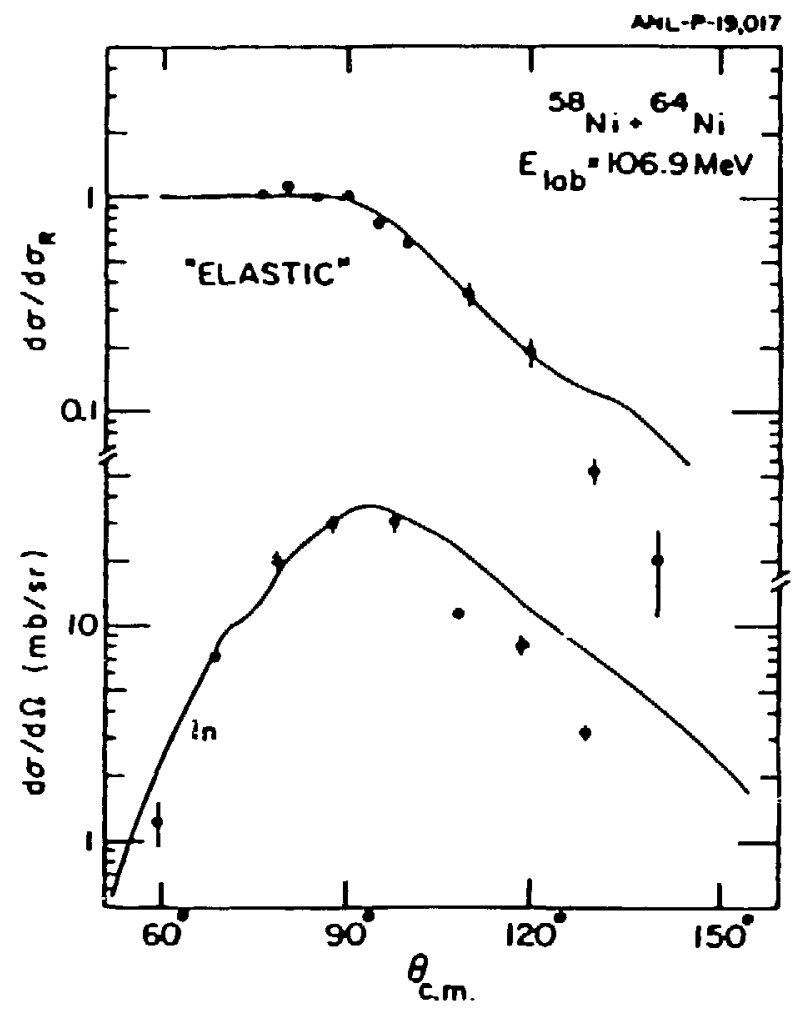

Figure 6. Quasi-elastic and one-particle transfer cross sections for ${ }^{68} \mathrm{Ni}+{ }^{64} \mathrm{Ni}$ compared to couples-channels calculations [18]. 
These coupled-channels calculations have an ingoing-wave boundary condition to describe the fusion and also allow for vibrational excitations to second-order. No imaginary potential is used. Some residual absorption could be introduced to improve the agreement with the data at the more backward engles. However, it is interesting to see how well the calculations reproduce the data without an imaginary potential.

The corresponding ${ }^{58} \mathrm{Ni}+{ }^{64} \mathrm{Ni}$ fusion calculations are shown in Figure 7 . The uppermost solid curve shows that although the relatively large one-particle cross sections are reproduced, their effect on top of the second-order vibrational couplings is not so strong and they do not change the low-energy slope. Allowing for a direct two-neutron transfer seems to be required, as indicated by the dashed curve.

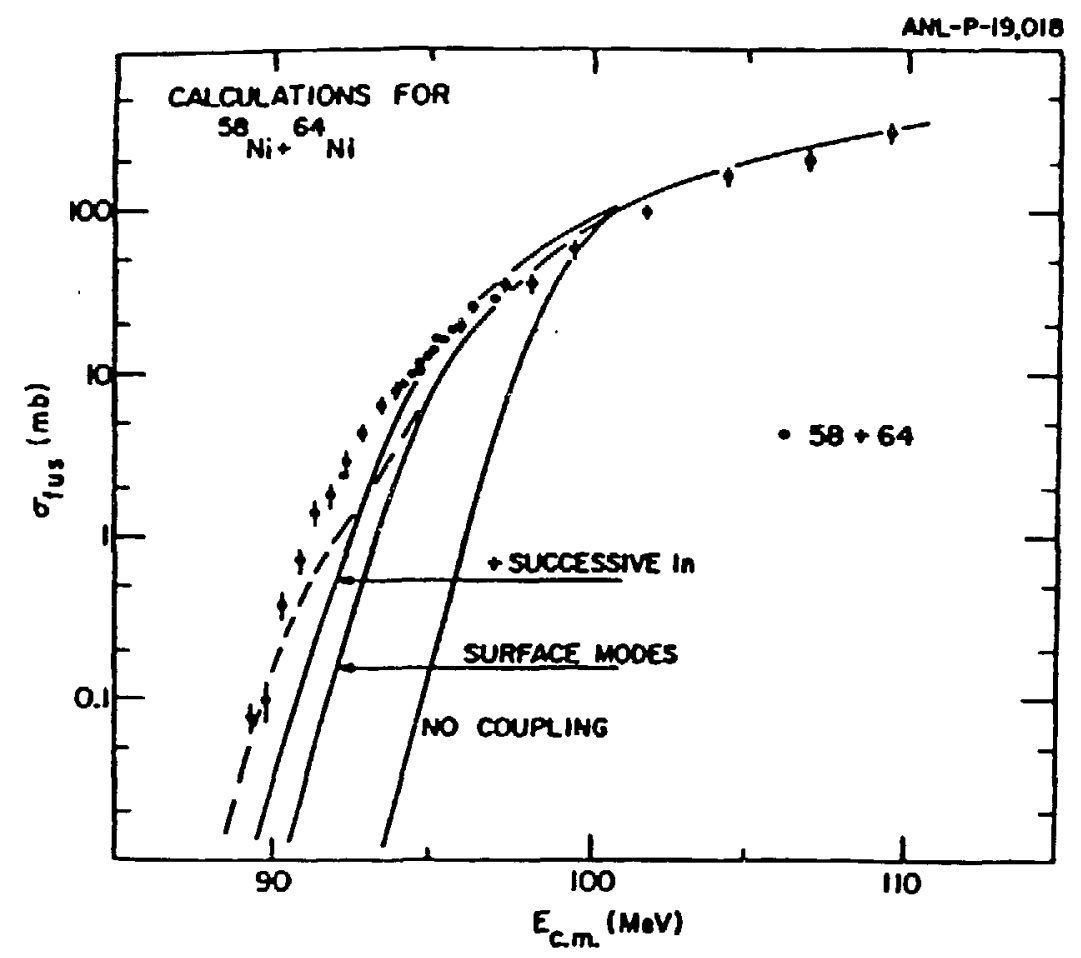

Figure 7. Fusion calculations for ${ }^{58} \mathrm{Ni}+{ }^{04} \mathrm{Ni}$. Ref. [18]

\section{SUB-BARRIER TRANSFER REACTIONS WITH DEFORMED NUCLEI}

The idea that transfer couplings depend on the distance between vibrating surfaces has a natural analogue in the case when permanently deformed nuclei collide. Here one would expect that the transfer should depend on the relative orientation of the nuclear surfaces. Consequently, the low energy transfer cross section for two well deformed prolate nuclei should be larger than for an equivalent spherical case. The curves in Figure 8 show some recent estimates for two-neutron transfer between different $\mathrm{Sm}$ isotopes [19]. The ${ }^{144} n_{3}$ is treated as a sphere and ${ }^{154} \mathrm{Sm}$ is taken to be deformed. A significant part of the differences shown in Figure 8 are due to nuclear sizes and reaction Q-values. These have to be taken into account in order to isolate the effect of deformation. 
This work was supported by the U. S. Department of Energy, Nuclear Physics Division, under contract W-31-109-ENG-38.

\section{REFERENCES}

(1) M. Beckerman, Phys. Rep. 129 (1985) 145.

[2] J. S. Lilley, B. R. Fu'ton, M. A. Nagarajan, I. J. Thompson and D. W. Banes, Phys. Lett. B151 (1985) 181.

[3] M. A. Nagarajan, C. Mahaux and G. R. Satchler, Phys. Rev. Lett. $\underline{54}$ (1985) 1136.

[4] A. M. Stefanini, D. Bonamini, A. Tivelli, G. Montagno'i, G. Fortuna, Y. Nagashima, S. Beghini, S. Signorini, A. DeRosa, G. Inglima, M. Sandoli, G. Cardella and F. Rizzo, Phys. Rev. Lett. 5o (1087) 285s.

[5] C. H. Dasso, S. Landowne, G. Pollarolo and A. Winitier, Nuci. Phys. A459 (1986) 134.

6] C. H. Dasso, G. Pollarolo and S. Landowne, Nucl. Phys. A443 (1985) 365.

7 S. Landowne, C. H. Dasso and G. Pollarolo, Phys. Lett. B1/8 (1986) 336.

8] C. S. Lim, M. D. Rebgetz, R. H. Spear, W. J. Vermeer, M. P. Fewell, B. A. Robson and R. Smith, Phys. Lett. B200 (1988) 263.

[9] M. A. Nagarajan and G. R. Satchler, Phys. Lett. B173 (1986) 29.

[10] C. H. Dasso, S. Landowne and A. Winther, Nucl. Phys. A405 (1983) 381; A407 (1983) 221.

[11] C. Y. Wong, Phys. Rev. Lett. 31 (1973) 766.

12] M. L. Halbert, J. R. Beene, D. C. Hensley, K. Honkanen, T. M. Semkow, V. Abenante, D. G. Sarantites and Z. Li, Sixth Adriatic International Conference on INuclear Physics, Dubrovnik, Yugoslavia (1987).

[13] C. H. Dasso, H. Fsobensen and S. Landowne, Phys. Rev. Lett. 57 (1986) 1498.

14] R. Vandenbosch, T. Murakami, C. C. Sahm, D. D. Leach, A. Ray and M. J. Murphy, Phys. Rev. Lett. 56 (1986) 1234.

[15] H. Esberisen and S. Landowne, Nucl. Phys. A467 (1987) 136.

16 H. Esbensen and S. Landowne, Phys. Rev. C 35 (1987) 2090.

17) K. E. Rehm, F. L. H. Wolfs, A. M. van den Berg and W. Henning, Phys. Rev. Lett. $55(1985) 280$.

18 H. Esbensen, Winter Workshop on Nuclear Dynamics V, Sun Valley, Idaho (1988).

19 S. Landowne and C. H. Dasso, Phys. Lett. B202 (1988)' 31.

20] F. L. H. Wolfs, W. Henning, K. E. Rehm and J. P. Schiffer, Phys. Lett. B196 (1987) 113.

\section{DISCLAIMER}

This report was prepared as an account of work sponsored by an agency of the United States Government. Neither the United State: Government nor any agency thereof, nor any of their employees, makes any warranty, express or implied, or assumes any legal liability or responsibility for the accuracy. completeness, or usefuiness of any information, apparatus, product, or process disclosed, or represents that its use would not infringe privately owned rights. Reference herein to any specific commercial product, proce, , s service by trade name, trademark, manufacturet, or otherwise does not necessarily cor-litute or imply its endorsement, recommendation, or favoring by the United States Goucrument or any agency thereof. The views and opinions of authors expressed herein do not necessarily state or feflect those of the United States Government or any aguncy thereof. 